\title{
Incident opioid drug use and adverse respiratory outcomes among older adults with COPD
}

\author{
To the Editor:
}

We read with great interest the observational, registry-based study of the association between an incident opioid prescription and risk of subsequent hospitalisation or death within 30 days in people aged 66 years or older in the community with a diagnosis of chronic obstructive pulmonary disease (COPD), published recently in the European Respiratory Journal [1]. The study was carefully conducted and accounts for potential confounding factors for which data were available. However, as with all observational studies, there are issues that affect interpretation of the findings and their application to clinical practice.

What does this study tell us? People with an incident opioid prescription had a 30-day mortality risk of $1.9 \%$ compared with $1.1 \%$ in people without an opioid prescription after propensity score matching; an absolute difference of $0.8 \%$ [1]. Importantly, stronger opioids were associated with lower risk than weaker opioids. Most opioids were prescribed short term in combination with acetaminophen or nonsteroid agents (about 90\%), almost certainly for musculoskeletal pain [1]. Patients in palliative care based on physician code were excluded because "the goals of care and indications for opioid use differ in the palliative care context". We disagree with this statement. The goals of opioid prescribing in palliative care and general medicine are the same: for the relief of symptoms (pain and breathlessness). It is difficult to see how this reduces potential confounding as access to palliative care services is not systematic for people with advanced disease, especially for those with nonmalignant disease, and thus would be a suboptimal marker for end-of-life care.

This study does not tell us that the observed risk difference is actually due to the opioid medication, as highlighted by a previous commentary [2]. In addition there are indications that the findings could be at least partly due to differences in underlying health status or other unmeasured factors, meaning that, despite the propensity score matching, people prescribed an opioid were more unwell. Firstly, the risk estimates varied markedly between sensitivity analyses with an almost $50 \%$ decrease in the excess risk in some models lessening the likelihood of the overall findings being clinically significant. We have no information about important factors such as the prevalence and severity of, or impairment due to, symptoms, health and functional status, the degree of clinical safety and effectiveness monitoring and why, how or at what dose the opioid was used. In addition, there does not appear to be any consideration of nonsteroidal anti-inflammatory drug (NSAID) use, which has been shown to increase mortality in COPD with ischaemic heart disease as a comorbidity [3]. Both the new opioid and control groups used in the present paper had a presence of ischaemic heart disease of more than $40 \%$, making NSAID use a potential confounder in the analyses [1]. Thirdly, the opioid group had higher 30-day mortality from cancer [1]. This is difficult to attribute to the opioid treatment.

Regular, oral, low-dose, extended-release morphine is the pharmacological treatment with the best evidence for relieving severe chronic breathlessness in people with advanced disease including COPD [4]. We know from randomised, prospective studies that these people have high risk of adverse events due to their severe illness but the evidence to date is that the risk of serious adverse events (respiratory depression, hospitalisation or death) does not seem to be increased by low-dose oral morphine in the short-term [4]. Future pooled analyses of randomised and pharmacovigilance data of opioids for breathlessness will further inform us about how to optimise the net clinical benefit for the individual

@ERSpublications

Extended-release morphine is underused for breathlessness. Careful titration will ensure better symptom control http://ow.ly/wtN03095IdM

Cite this article as: Ekström M, Bajwah S, Johnson MJ. Incident opioid drug use and adverse respiratory outcomes among older adults with COPD. Eur Respir J 2017; 49: 1602311 [https://doi.org/10.1183/ 13993003.02311-2016]. 
patient and about the longer-term effects. Safe and effective treatment of chronic breathlessness using regular, low-dose oral morphine requires that the right dose is used for the right patient for the right level of symptom severity in the context of good clinical follow-up [4]. Currently, low-dose extended-release morphine is likely underused for breathlessness in very severe COPD [5]. Careful titration will ensure better symptom control for a prevalent and frightening symptom that people experience over long periods in advanced disease towards the end of life.

Magnus Ekström $^{1}$, Sabrina Bajwah ${ }^{2}$ and Miriam J. Johnson ${ }^{3}$

${ }^{1}$ Department of Clinical Sciences, Division of Respiratory Medicine \& Allergology, Lund University, Lund, Sweden.

${ }^{2}$ Cicely Saunders Institute, King's College London, London, UK. ${ }^{3}$ Hull York Medical School, University of Hull, Hull, UK.

Correspondence: Sabrina Bajwah, Cicely Saunders Institute, King's College London, London, UK.

E-mail: sabrina.bajwah@kcl.ac.uk

Received: Nov 242016 | Accepted: Dec 112016

Conflict of interest: None declared.

\section{References}

1 Vozoris NT, Wang X, Fischer HD, et al. Incident opioid drug use and adverse respiratory outcomes among older adults with COPD. Eur Respir J 2016; 48: 683-693.

2 Downar J, Colman R, Horton R, et al. Opioids in COPD: a cause of death or a marker of illness severity? Eur Respir J 2016; 48: 1520-1521.

3 Antman EM, Bennett JS, Daugherty A, et al. Use of nonsteroidal antiinflammatory drugs. An update for clinicians: a scientific statement from the American Heart Association. Circulation 2007; 115: 1634-1642.

4 Ekström M, Nilsson F, Abernethy AA, et al. Effects of opioids on breathlessness and exercise capacity in chronic obstructive pulmonary disease: a systematic review. Ann Am Thorac Soc 2015; 12: 1079-1092.

5 Ahmadi Z, Bernelid E, Currow DC, et al. Prescription of opioids for breathlessness in end-stage COPD: a national population-based study. Int J Chron Obstruct Pulmon Dis 2016; 11: 2651-2657.

\section{Incident opioid use is associated with risk of respiratory harm in non-palliative COPD}

\section{From the authors:}

We thank M. Ekström and co-workers for their interest in our manuscript [1] and for their contributions to the important topic of opioid drug use in chronic obstructive pulmonary disease (COPD). M. Ekström and co-workers stress the efficacy of selective use of opioids for refractory breathlessness in COPD. We acknowledged in our manuscript [1] that several clinical studies demonstrate that opioids are efficacious in selected COPD patients for relieving refractory breathlessness. However, we have previously outlined reasons why such trials of drug efficacy have limited ability to comprehensively evaluate for possible drug harms [1-3]. Furthermore, chronic musculoskeletal pain, and not respiratory symptoms, is likely the more common reason why individuals with COPD use opioid drugs [3, 4]. Indeed, M. Ekström and co-workers recently published data showing that $97 \%$ of opioid prescriptions among Swedes with advanced COPD were for pain [5]. Several Cochrane reviews have suggested there is insufficient evidence to support the use of opioids for chronic musculoskeletal pain [6-8].

We would like to clarify and correct several points that M. Ekström and co-workers made regarding our study. First, the authors appear to focus on one result, that the absolute risk of all-cause mortality was only $0.8 \%$ higher among community-dwelling incident opioid users versus non-users. Although this

@ERSpublications

The potential for respiratory-related harm needs to be considered when using opioid drugs in nonpalliative COPD http://ow.ly/i5W03095MKV

Cite this article as: Vozoris NT, O'Donnell DE, Bell C, et al. Incident opioid use is associated with risk of respiratory harm in non-palliative COPD. Eur Respir J 2017; 49: 1602529 [https://doi.org/10.1183/ 13993003.02529-2016]. 
specific risk difference is small, it may be clinically important at a population level. Furthermore, the absolute risk difference for all-cause mortality between the exposed and control groups was larger when one considered use of opioid-only formulations in the community (3.6\%; number needed to harm $(\mathrm{NNH})$ 28) or opioid use among long-term care residents (9.3\%; NNH 11) [1].

Second, the authors wrote that "stronger opioids were associated with lower risk than weaker opioids". We reported the opposite. Use of more potent opioid-only agents was associated with greater respiratory-related morbidity and mortality than use of the less potent combination opioid/non-opioid agents (see table 4 in [1]).

Third, Ekstrom et al. wrote "the risk estimates varied markedly between sensitivity analyses with an almost $50 \%$ decrease in the excess risk in some models". This statement is not accurate. Our sensitivity analyses showed an overall pattern of increased risk for adverse events among opioid users, regardless of opioid half-life duration, opioid dose and COPD severity.

Fourth, the authors propose that the increased adverse respiratory event risk associated with incident opioid use may be due to unmeasured factors not included in our propensity score model, like respiratory symptoms, general health status, and opioid indication and dose. While we do not have data on respiratory symptoms in our databases, we incorporated other markers of COPD severity in our propensity score model, the most important of which was COPD exacerbation frequency, which is known to be associated with quality of life [9]. We also included multiple markers of general health status in our propensity score model, including nine different comorbidities. Opioid indication and dose could not be included in the propensity score model, because these variables apply only to the exposed and not the control group. Instead, we conducted a sensitivity analysis by COPD severity and found increased mortality associated with opioid use among the healthiest subgroup of individuals and this subgroup would be the least likely to receive opioids for respiratory symptoms (see table 7 in [1]). We also conducted an opioid dosing sensitivity analysis (see table 6 in [1]).

Fifth, we acknowledge that we did not incorporate nonsteroidal anti-inflammatory drug (NSAID) use in our propensity score model and this could have resulted in residual confounding. We included the presence of musculoskeletal and connective tissue diseases in our propensity score model (individuals with these comorbidities would be the ones more likely to use NSAIDs) and the exposed and control groups were well balanced on this variable. Furthermore, while concomitant NSAID use among opioid recipients may have contributed to the finding of increased all-cause mortality via cardiac ischaemia or gastrointestinal tract bleeding, we fail to see how NSAID use would explain the higher risks of outpatient respiratory exacerbations and COPD or pneumonia-related emergency room visits and hospitalisations among opioid users.

Finally, M. Ekström and co-workers point out that cancer deaths were higher in the exposed versus control group in our study. While this is correct, we mentioned in our manuscript that we repeated our analysis, excluding individuals with lung cancer and any cancer, and we still observed increased respiratory-related morbidity and mortality with incident opioid use (see online supplement 1 in [1]).

We agree with a concluding comment by M. Ekström and co-workers that "pooled analyses of randomised and pharmacovigilence data" on opioids are needed to optimally inform clinical practice, as this approach combines the best data on drug efficacy with the best data on drug harms [10].

Nicholas T. Vozoris ${ }^{1,2,3}$, Denis E. O’Donnell ${ }^{4}$, Chaim Bell ${ }^{3,5,6,7}$, Sudeep S. Gill ${ }^{4,5}$ and Paula A. Rochon ${ }^{3,5,6,8}$

${ }^{1}$ Division of Respirology, Department of Medicine, St. Michael's Hospital, Toronto, Ontario, Canada. ${ }^{2}$ Keenan Research Centre in the Li Ka Shing Knowledge Institute, St Michael's Hospital, Toronto, Ontario, Canada. ${ }^{3}$ Department of Medicine, University of Toronto, Toronto, Ontario, Canada. ${ }^{4}$ Department of Medicine, Queen's University, Kingston, Ontario, Canada. ${ }^{5}$ Institute for Clinical Evaluative Sciences, Toronto, Ontario, Canada. ${ }^{6}$ Institute of Health Policy, Management, and Evaluation, University of Toronto, Toronto, Ontario, Canada. ${ }^{7}$ Division of General Internal Medicine, Department of Medicine, Mount Sinai Hospital, Toronto, Ontario, Canada. ${ }^{8}$ Women's College Research Institute, Women's College Hospital, Toronto, Ontario, Canada.

Correspondence: Dr. Nicholas Vozoris, Division of Respirology, Department of Medicine, St. Michael's Hospital, 30 Bond Street, Toronto, Ontario, Canada, M5B 1W8. E-mail: nick.vozoris@utoronto.ca

Received: Dec 232016 | Accepted: Dec 232016

Conflict of interest: Disclosures can be found alongside this article at erj.ersjournals.com

\section{References}

1 Vozoris NT, Wang X, Fischer HD, et al. Incident opioid drug use and adverse respiratory outcomes among older adults with COPD. Eur Respir J 2016; 48: 683-693.

2 Vozoris NT, O’Donnell DE, Bell CM, et al. Opioids in COPD: a cause of death or a marker of illness severity? Eur Respir J 2016; 48: 1521-1522. 
3 Vozoris NT, O'Donnell DE. The need to address increasing opioid use in elderly COPD patients. Expert Rev Respir Med 2016; 10: 245-248.

4 Vozoris NT, Wang X, Fischer HD, et al. Incident opioid drug use among older adults with chronic obstructive pulmonary disease: a population-based cohort study. Br J Clin Pharmacol 2016; 81: 161-170.

5 Ahmadi Z, Bernelid E, Currow DC, et al. Prescription of opioids for breathlessness in end-stage COPD: a national population-based study. Int J Chron Obstruct Pulmon Dis 2016; 11: 2651-2657.

6 Noble M, Treadwell JR, Tregear SJ, et al. Long-term opioid management for chronic noncancer pain. Cochrane Database Syst Rev 2010; CD006605.

7 Chaparro LE, Furlan AD, Deshpande A, et al. Opioids compared to placebo or other treatments for chronic low-back pain. Cochrane Database Syst Rev 2013; 8: CD004959.

8 Gaskell H, Moore RA, Derry S, et al. Oxycodone for neuropathic pain and fibromyalgia in adults. Cochrane Database Syst Rev 2014; 6: CD010692.

9 Spencer S, Calverley PM, Burge PS, et al. Impact of preventing exacerbations on deterioration of health status in COPD. Eur Respir J 2004; 23: 698-702.

10 Vandenbroucke JP, Psaty BM. Benefits and risks of drug treatments: how to combine the best evidence on benefits with the best data about adverse effects. JAMA 2008; 300: 2417-2419. 